\title{
Enfermedad de Pompe: reporte de caso
}

\author{
Disease Pompe: report of case \\ Sara Ariass ${ }^{1}$, Mariana Gómez ${ }^{1}$, Isabel Fernández, Luis Gustavo Celis ${ }^{1}$
}

\section{Resumen}

Objetivo. Se describe el caso de un paciente masculino de siete meses de edad, evaluado por cardiología a los quince días de vida por antecedente de muerte súbita cardíaca de hermana a los cuatro meses, no se sospechó nada pese a consanguinidad de padres y diagnóstico temprano del paciente de cardiomiopatía. Evoluciona con un cuadro clínico de infecciones respiratorias a repetición desde los tres meses (bronquiolitis recurrente), falla de medro y cuadro de neumonía reciente y fallece a los ocho días de la consulta con la genetista. Métodos. El abordaje inicial fue la realización de un ecocardiograma a los quince días de nacido por antecedente de muerte súbita de hermana a los cuatro meses por cardiomegalia y consanguinidad de los padres. Continúa con deterioro clínico a través de los meses por lo que se remite a genética, se toman pruebas enzimáticas en gota de sangre seca, el paciente fallece antes de recibir el diagnóstico de Enfermedad de Pompe. Resultados. Se enfocó al paciente con un posible diagnóstico de Enfermedad de Pompe solicitándose enzima lisosomal alfaglucosidasa (GAA) en muestra de gota de sangre seca y reporte final de la secuenciación genética.

Palabras Clave: Enfermedad de Pompe, GAA ( $\alpha-1,4$ glucosidasa lisosomal (maltasa ácida), consanguinidad, cardiomiopatía, hipotonía.

\section{Abstract \\ Objective. It is a case of a seven months male patient, evaluated by cardiology fifteen days after he was born secondary, to sudden cardiac death of his sister of four months, nothing suspicious despite consanguinity of parents and early diagnosis of the patient with Cardiomyopathy. Evolved with the following clinical conditions recurrent respiratory infections from three months (recurrent bronchiolitis), widespread malnutrition and recent pneumonia. Died eight days after the consultation with the geneticist. Methods. The initial approach was to perform echocardiogram at fifteen days old, because of history of the sudden death of his sister of four months, cardiomegaly and parental consanguinity. Clinical deterioration continues through months so he is referred to genetic, enzymatic tests, taken in dried blood, the patient dies before receiving the diagnosis of Pompe disease. Results. The patient was focused with a possible diagnosis of Pompe Disease so lysosomal enzyme $\alpha$-glucosidase (GAA) sample was requested in dried blood and genetic sequencing final report to define diagnosis.}

Keywords: Pompe disease, GAA ( $\alpha-1.4$ lysosomal glucosidase (acid maltase), consanguinity, cardiomyopathy, hypotonia. 


\section{Introducción}

La enfermedad de Pompe (EP) (enfermedad de depósito de glucógeno tipo II o "déficit de ácido maltasa"), fue descrita en 1932 por el patólogo holandés Johannes C. Pompe quien realizó la descripción de la enfermedad. Sin embargo fue hasta el 2006 cuando se aprobó en Estados Unidos y Europa la terapia de reemplazo enzimático, que modificó el curso de la enfermedad, y es cuando se prestó más importancia como enfermedad (1-3).

La EP es un raro trastorno neuromuscular, progresivo, de instauración rápida, debilitante e incluso mortal $(1,2)$, causada por el déficit de la enzima lisosomal alfa-glucosidasa (GAA), con manifestaciones clínicas variables lo cual representa un desafío diagnóstico y retrasa su tratamiento (4).

La EP hace parte de las enfermedades huérfanas o raras que dependiendo de la zona se definen en el Acta de Enfermedades Raras del 2002 como cualquier enfermedad o condición que afecte a menos de 200.000 personas en los Estados Unidos, siendo equivalente a 1:500 personas, o según la Unión Europea como aquellas de origen genético o adquirido, con peligro de muerte o invalidez crónica y cuya prevalencia sea inferior a 1 en 2000 individuos $(5,6)$.

Dentro de este grupo se encuentran las enfermedades por depósito lisosomal (caracterizadas por el depósito de compuestos naturales en pequeñas vesículas de las células que afectan diversos órganos), siendo enfermedades escasas aproximadamente podrían llegar a ser 50 enfermedades en este grupo como la Mucopolisacaridosis Tipo I y II (MPS), Enfermedad de Gaucher, Enfermedad de Fabry, entre otras; donde la EP hace parte de uno de los trastornos de mayor incidencia. Estas enfermedades se caracterizan por acumulación de compuestos no degradados en órganos o tejidos que generan su deterioro y disfuncionalidad, así mismo la excreción de sustancias en la orina. $(1,3)$

\section{Reporte de caso}

Se presenta el caso de un paciente masculino lactante menor de siete meses de edad valorado en consulta externa de genética en Caracas-Venezuela por cuadro clínico de infecciones respiratorias a repetición desde los tres meses (bronquiolitis recurrente), falla de medro y cuadro de neumonía reciente. Al examen físico se encuentra sin control cefálico, peso de 5.700 gramos, hipotonía generalizada, déficit pondoestatural, paladar grueso y fascias atípicas, que pueden ser evidenciado en las Figuras 1 y 2, las cuales se tomaron previo consentimiento informado. Ocho días después el paciente fallece por cuadro de dificultad respiratoria aguda sin control de la misma.
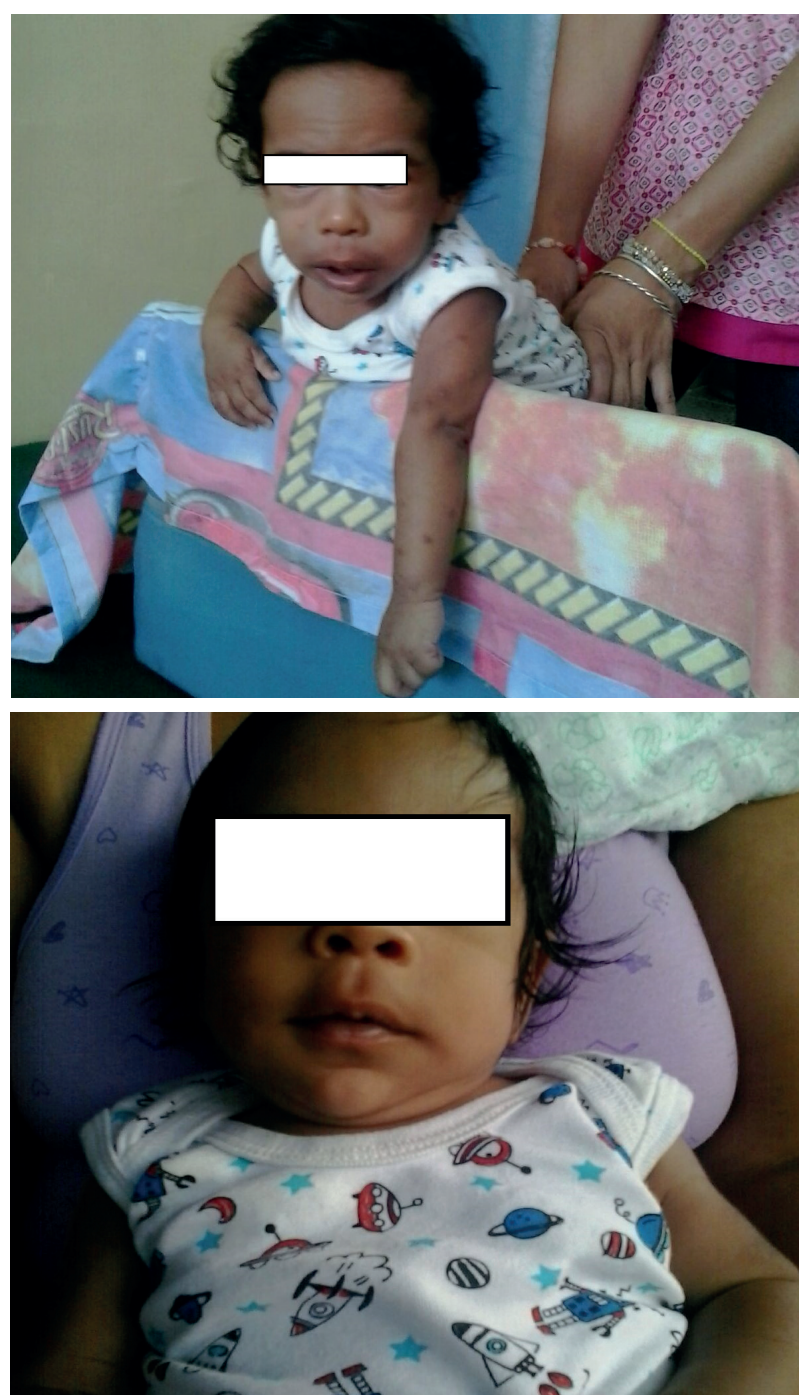

Figuras 1 y 2. Muestran el deterioro progresivo del paciente. 
Como antecedentes de importancia padres consanguíneos, primos hermanos, producto de tercer embarazo por parto vaginal, a término, eutócico, vigoroso. Peso de 3.450 gramos y talla de $50 \mathrm{~cm}$, considerados adecuados para edad gestacional. Llama la atención muerte cardiaca súbita de hermana a los cuatro meses de edad (segundo embarazo a término).
El paciente por los antecedentes comentados anteriormente es valorado a los quince días por cardiología donde evidencian una cardiomiopatía hipertrófica del ventrículo izquierdo, la cual manejan con espironolactona y propanolol, no se realizan estudios de extensión.

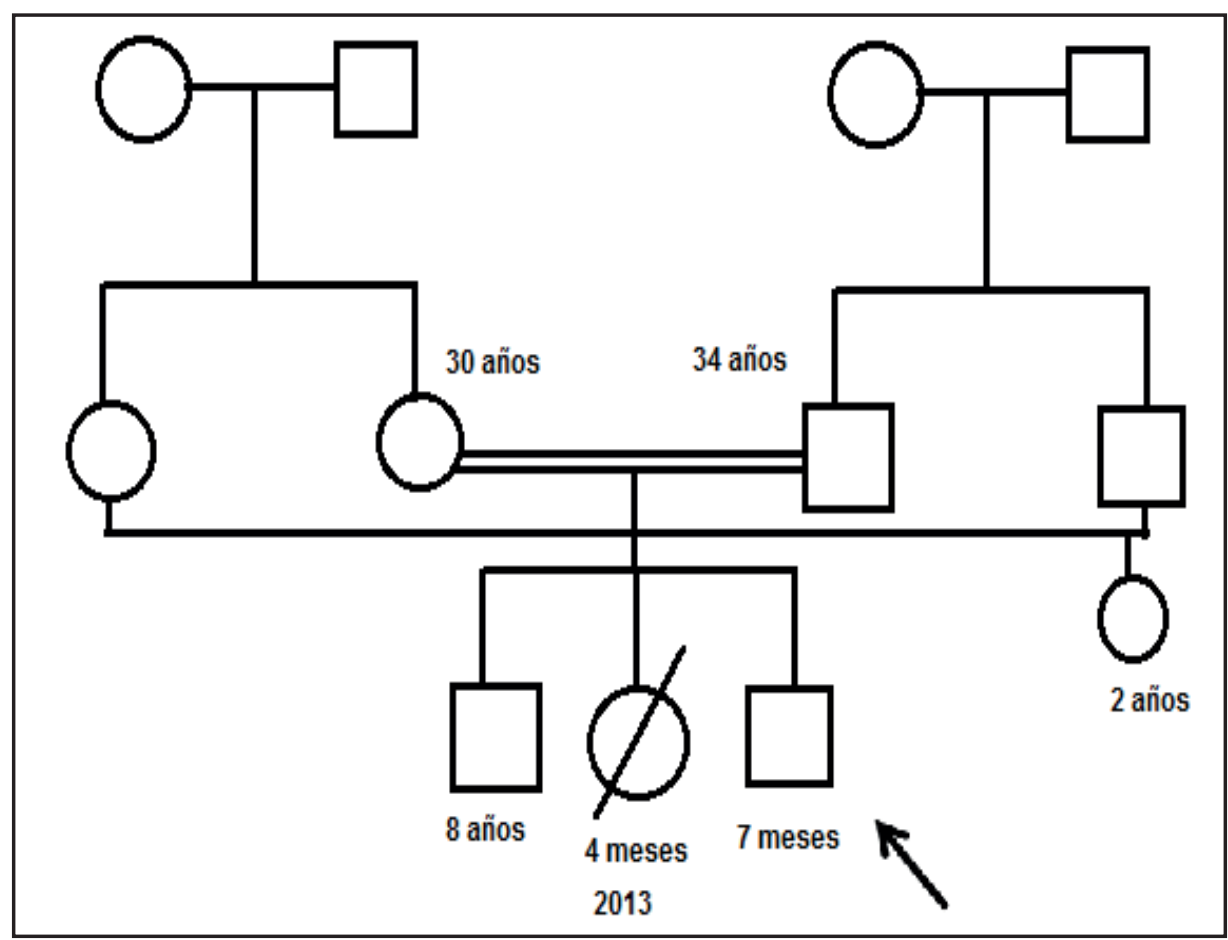

Figura 3. Genealogía del paciente en estudio.

En el momento de la consulta se realiza un cariotipo, el que era normal 46, XY; a su vez se toma una muestra sanguínea en papel de filtro para determinación enzimática de ácido alfa glucosidasa. Los exámenes llegaron nueve días después de fallecido el paciente que confirmaban la Enfermedad de Pompe, Tabla 1. Estos resultados señalan que la actividad de la ácido alfa glucosidasa en esta muestra está dentro de los límites para establecer diagnóstico. Sin embargo, no se puede excluir la presencia de una pseudodeficiencia de alelos.

Al recibir los resultados, se estudia un hermano primogénito de ocho años para descartar déficit parcial de la enzima con determinación enzimática la cual presenta unos niveles normales, al igual se estudia prima en primer nivel de dos años de padres consanguíneos que también descartan la patología. 
Tabla 1. Resultados enzimáticos en muestra de gota de sangre seca. Se aprecia una disminución significativa de la actividad enzimática.

\begin{tabular}{|c|c|c|c|c|}
\hline Desorden & Código & Resultado & $\begin{array}{c}\text { Valor de } \\
\text { Referencia }\end{array}$ & Unidades \\
\hline $\begin{array}{c}\text { Pompe } \\
\text { (Acido alfa } \\
\text { glucosidasa) }\end{array}$ & GAA & 0.07 & +4.46 & umol/L/h \\
\hline
\end{tabular}

Posteriormente se realizaron pruebas moleculares para confirmar el diagnóstico y caracterizar la mutación del paciente en estudio que fue realizada en el Boston Children's Hospital, Tabla 2.

Tabla 2. Reporte final de la secuenciación.

\begin{tabular}{|c|c|c|c|}
\hline Clasificación & Cigocidad & $\begin{array}{c}\text { Cambio } \\
\text { nucleótido }\end{array}$ & $\begin{array}{c}\text { Efecto de la } \\
\text { proteína/Cambio } \\
\text { del aminoácido }\end{array}$ \\
\hline Patógeno & Homocigote & c.2237G >A & p.Trp746Ter \\
\hline
\end{tabular}

\section{Discusión}

La EP es un depósito lisosomal anormal de glucógeno debido al déficit o ausencia de GAA en múltiples tejidos, principalmente con compromiso cardíaco y esquelético (3).

En cuanto a datos epidemiológicos, hasta el 2011 se encuentran 742 pacientes en el Registro Internacional de la Enfermedad de Pompe, con casos provenientes de 28 paises $(1,2)$. No se encuentran datos epidemiológicos con respecto a la EP en Latinoamérica. Con respecto a diferencias geográficas se estima que la incidencia de EP temprana en afroamericanos, chinos y holandeses es mayor con respecto al resto de la población mundial $(1,6,7)$.

La causa de la EP es la deficiencia de $\alpha-1,4$ glucosidasa lisosomal (maltasa ácida), que provoca una acumulación del glucógeno lisosomal en múltiples tejidos y células, con afectación primordial del músculo esquelético, músculo cardiaco y la musculatura respiratoria, que en el contexto clínico del paciente se manifiesta con la debilidad muscular, cardiomiopatía, deterioro de la función respiratoria y alteración del músculo liso con manifestaciones gastrointestinales $(1,4)$.

La enfermedad se hereda de forma autosómica recesiva de tal forma que son importantes antecedentes de consanguinidad o endogamia, como en el caso de nuestro paciente, lo cual ayudó a orientar el diagnóstico. De acuerdo a la herencia, en el momento de la concepción entre parientes con grado de consanguinidad cercano cada hijo puede verse afectado hasta el $25 \%, 50 \%$ de ser un portador asintomático y un $25 \%$ de ser sano.

El gen relacionado con esta patología se localiza en el locus 17q25.2-q25.3. Se han encontrado numerosos cambios en la secuencia, de los cuales $75 \%$ son patógenos, lo cual puede afectar el mecanismo de corte y empalme de los exones, la estabilidad del ARN mensajero y biosíntesis de la proteína ocasionando que la enzima no actue. Deseamos resaltar que en el caso del paciente estudiado, se ha encontrado una variante que no ha sido reportada en la literatura, por sus características de homocigocidad (8). Este diagnóstico molecular puede tener dificultades en la interpretación. Sin embargo es un valioso aporte en la realización del asesoramiento genético y plantea la posibilidad de realizar pruebas de diagnóstico prenatal, permitiendo la orientación sobre los pasos a tomar una vez la mujer queda embarazada y se debe tener en cuenta la importancia del diagnóstico preconcepcional que previene y evita daños irreversibles en el infante $(9,10)$.

Uno de los posibles factores responsables por la amplia variabilidad clínica se encontró recientemente y ha sido el polimorfismo en la deleción en el gen que codifica para la enzima convertidora de angiotensina (ECA), que se ha visto aumenta el número de fibras tipo II e influencia las propiedades musculares, asociado con un inicio temprano y altos niveles de creatin-cinasa (3).

Los lisosomas son organelos conformados por retículo endoplasmático rugoso y luego empaquetados por el aparato de Golgi con enzimas hidrolíticas y 
proteolíticas encargados de reciclar diferentes organelos de la célula y liberar los residuos en el citosol. En este grupo de enfermedades fisiopatológicamente se producen una incapacidad de degradar moléculas por un déficit enzimático específico de una hidrolasa (GAA), de una proteína activadora de la enzima o de un transportador de la membrana lisosomal, que se encarga de facilitar la salida de pequeñas moléculas hacia el exterior del organelo. La función de la GAA es hidrolizar la maltosa, oligosacáridos lineales y cadenas externas de glucógeno para obtener glucosa; esta enzima se encuentra en todos los tejidos (3).

Lo anterior genera una lesión primaria caracterizada por el acúmulo progresivo de glucógeno intralisosomal, alterando el proceso de autofagia, es decir, la degradación de diferentes componentes del citoplasma que favorece el balance entre su biogénesis y su degradación. Este acúmulo excesivo de glucógeno en el lisosoma puede llegar a ocasionar ruptura de membranas lisosomales y posterior daño en el aparato contráctil de fibras musculares como fibras miocárdicas, musculares y lisas (3).

Al conocer la fisiopatología de la EP se puede esperar determinadas manifestaciones clínicas importantes para tener en cuenta en el diagnóstico de esta enfermedad ya que afecta a todos los tejidos y órganos.

La EP manifiesta hallazgos clínicos diversos, por lo cual se recurre a pautas de diagnóstico y tratamiento del American College of Medical Genetics que la clasifica en dos variedades clínicas:

1. Temprana o clásica: inicia en los primeros doce meses de vida y es la forma grave de la enfermedad con dificultad para alimentación, infecciones respiratorias recurrentes, falla en el medro, poca movilidad e hipotonía; con una afección cardiaca más severa y cuyo desenlace mayormente es la muerte. Dentro de los hallazgos que conforman la triada clínica están: hipotonía, hepatomegalia y cardiomegalia $(2,6,10)$.

2. Tardía: inicia luego del año de vida, caracterizada por debilidad proximal de los miembros inferiores $\mathrm{y}$ músculos paraespinales, afección diafragmática y de los músculos accesorios respiratorios que puede progresar a apnea obstructiva del sueño progresiva y falla respiratoria crónica. Cuentan con mejor pronóstico, sin embargo su diagnóstico es más difícil $(10,11)$.

En el caso de nuestro paciente clínicamente es una EP de forma temprana debido a la edad del paciente, las características de la triada clásica como la hipotonía que en el examen físico se evidenció como una disminución del tono muscular progresivo, retraso en la adquisición del sostén cefálico, sin desarrollar la sedestación; en cuanto a la hepatomegalia, no se documenta en nuestro caso pero se debe tener en cuenta que es detectable desde el mes de vida y puede llegar a ser moderada; y la cardiomegalia que en este paciente se documenta como un hallazgo ecocardiográfico de cardiomiopatía hipertrófica del ventrículo izquierdo, lo que concuerda con los observaciones de la literatura del producto de la obstrucción en el flujo de salida del ventrículo izquierdo, provocando falla cardiaca antes de los ocho meses de vida.

Otros hallazgos importantes que se tienen que tener en cuenta son:

- Trastornos en las motoneuronas de la médula y núcleos del tallo generando la debilidad muscular severa de estos pacientes afectando músculos más próximos, que distales. Complicaciones como contracturas, deformidades de extremidades y del tronco y osteopenia/osteoporosis $(11,12)$.

- Macroglosia, debilidad de la lengua

- Anomalías auriculares reflejando posiblemente una patología coclear o conductiva

- Problemas de la alimentación, principalmente de succión y de deglución por debilidad de la lengua y de la musculatura facial, que están relacionados con dificultad en la ganancia ponderal.

- Dificultad respiratoria, por afectación con neumonías recurrentes, asociada a la debilidad e hipotonía del diafragma y músculos intercostales que llevan a una disminución del reflejo tusígeno, lo que incrementa la susceptibilidad a infecciones y broncoaspiración (11). 
La causa principal de la enfermedad se da por el déficit de GAA, gracias a los hallazgos paraclínicos en los diferentes tejidos. Se describe la actividad enzimática medida en linfocitos, la cuantificación de la actividad de la GAA mediante nuevas técnicas (con alta sensibilidad y especificidad) y la actividad enzimática de la GAA medida en fibroblastos como el gold estándar, debido a que los fibroblastos cultivados de la piel tienen la mayor actividad de GAA y muy poca actividad de otras enzimas como \- glucosidasa neutra por lo cual la interferencia es mínima, sin embargo el resultado tarda de 4 a 6 semanas, es una técnica invasiva y que no se realiza en nuestro medio $(4,12)$.

Igualmente, puede realizarse biopsia muscular que evidencian vacuolas positivas con la prueba del ácido peryódico de Schiff que identifica al glucógeno depositado dentro del lisosoma (12).

Ante un cuadro sugestivo de EP tenemos paraclínicos que complementarán el estudio de la enfermedad como lo son:

- Elevación de la creatina fosfocinasa (CPK) siendo un marcador inespecífico pero en la EP clásica puede elevarse hasta 2000 UI/L. Se debe tener en cuenta que se puede elevar en otras condiciones que deben ser consideradas $(1,10)$.

- Actividad enzimática de GAA: se puede realizar en muestras de sangre seca lo que permite el análisis rápido y sensible, se habla de un déficit completo (actividad $<1 \%$ de los controles normales) de la actividad enzimatica de GAA propio de la forma clásica de la enfermedad de Pompe; y de un déficit parcial (actividad 2\%-40\% de los controles normales) de la actividad enzimática asociada a las formas de inicio tardío $(10,12)$.

- Elevación de enzimas aspartato-aminotransferasa, alanina-aminotransferasa o la deshidrogenasa láctica al liberarse del músculo afectado.

- Radiografía de tórax con evidencia de cardiomegalia.

- Electrocardiograma con complejo QRS de alto voltaje y acortamiento del segmento PR.
- Electromiografía con evidencia de patrón miopático, descargas pseudomiotónicas (descargas miotónicas sin miotonía clínica), fibrilaciones, con velocidad de conducción motora y sensitiva normal.

- Test prenatales: test genéticos moleculares en busca de un gen específico ante hallazgos familiares; test genéticos bioquímicos de la medición de la actividad de la GAA en las vellosidades coriónicas o amniocitos (9-14).

Se deben considerar diferentes entidades basándonos en la clínica del paciente. En el caso de la hipotonía se puede contemplar enfermedades como la atrofia muscular espinal, miopatías metabólicas o congénitas, que se pueden diferenciar por la ausencia de la cardiomegalia $(1,10)$.

Por otro lado, en cuanto a la cardiopatía, el déficit de citocromo C oxidasa, glucogenolisis VIII o tipo IV podrían ser diagnósticos diferenciales.

Una de las entidades que cuenta con mayor similitud con la EP es la distrofia muscular de Duchenne que cuenta con pseudohipertrofia de los gemelos y debilidad proximal, la historia familiar materna positiva puede ayudar a diferenciar la distrofia muscular de Duchenne. La afección exclusiva en varones y los valores de CPK se dan de cuatro a cinco veces más que en la EP y en la electromiografía de la distrofia muscular de Duchenne no hay descargas miotónicas $(1,10)$.

La EP es una enfermedad que compromete varios sistemas, por lo cual se debe hacer un abordaje integral y multidisciplinario del paciente. Se deben incluir un audiólogo, cardiólogo, gastroenterólogo, genetista, neumólogo, neurólogo, nutriólogo, médico rehabilitador, ortopedista, otorrinolaringólogo y terapistas respiratorios, físicos, ocupacionales y de lenguaje, que se precisarán de acuerdo a las necesidades del paciente (12).

Así mismo es claro que el tratamiento de los infantes debe empezar en los primeros días 
después del nacimiento, no meses, pues entre más temprano inicie tratamiento, mayores ventajas se pueden contemplar $(2,3)$.

Se plantea la terapia de reemplazo enzimático como una estrategia de vital importancia para mejorar las funciones cardíacas al reducir la cardiomegalia; las funciones respiratorias, al disminuir los requerimientos de apoyo ventilatorio; y las funciones motoras. De este modo se puede aumentar la supervivencia cuando esta terapia se instaura oportunamente. Esta terapia consiste en la administración intravenosa de una forma precursora de la enzima $\alpha-1,4$ glucosidasa, que es capaz de penetrar en los lisosomas, en los pacientes con EP tardía tienen una respuesta adecuada y con la forma clásica se obtiene mejoría a corto plazo (12).

Para el manejo multidisciplinario se puede plantear un enfoque por sistemas así $(9,10,15)$ :

- Cardiológica: manejo conjunto con cardiólogo pediatra, ecocardiograma para determinar el grado de involucró del músculo cardiaco e intervenciones necesarias. Si tiene disfunción ventricular se puede usar digoxina, inotrópicos y diuréticos. Se ha reportado muerte súbita con el tratamiento con betabloqueadores, por lo que su uso debe ser cuidadoso (16 -18).

- Pulmonar: se evalúa con alteraciones del patrón respiratorio, fatiga y dificultad para la alimentación, con oximetría de pulso, gasometría para medir cantidad de $\mathrm{O} 2$ y CO2, y cuantificación de la capacidad vital y cuando sea posible el uso de una espirometría. $\mathrm{Al}$ ser propensos a infecciones pulmonares se debe utilizar antibióticos y broncodilatadores y terapia pulmonar para mantener la vía aérea limpia, se debe tratar la hipoxia o hipoventilación diurna y nocturna con oxígeno suplementario o considerar el uso continuo de presión positiva.

- Gastrointestinal/nutricional: se indica una videofluoroscopia, e inicio de alimentación gastroyeyunal o nasoduodenal ante la presencia de reflujo gastroesofágico, se debe asegurar una ingesta adecuada de calorías, vitaminas y minerales con una dieta rica en proteínas, baja en carbohidratos y ejercicio aeróbico con el objetivo de disminuir el acúmulo de glucógeno y compensar la oxidación de aminoácidos (10).

- Neurología: revisión de la función motora periódicamente (ya que el acúmulo de glucógeno afecta al cerebro), estudios de timpanometría, audiometría, potenciales evocados auditivos o emisiones otoacústicas.

- Osteomuscular: inicio oportuno de rehabilitación física, respiratoria ocupacional.

Así mismo se deben evitar posibles complicaciones tratando las infecciones adecuadamente, programa de vacunación adecuado para la edad, así como programa anual de vacunación del niño y de su familia contra la influenza, profilaxis para el virus sincitial respiratorio, y tener en cuenta que la anestesia se debe utilizar en el paciente solo cuando sea absolutamente necesario debido a que reduce el retorno cardiovascular y produce una posible insuficiencia respiratoria subyacente (10).

En cuanto a la educación a los padres existen programa de consejería genética donde se les da información adecuada para ayudarles a aceptar la historia natural de la enfermedad, y asesoría en decisiones que tenga que tomar la familia $(10,15)$.

\section{Conclusiones}

Es una enfermedad huérfana o rara, no por esto hay que dejarla de lado. Es importante conocerla ya que si no conoce no se diagnostica, su abordaje tiene que ser multidisciplinario para lograr mejores resultados. El papel del cardiólogo y pediatra son de mucha importancia ya que son los que dan los primeros pasos para lograr diagnóstico y tratamiento oportunos.

La información utilizada para la elaboración de este artículo ha contado con el permiso de la Unidad de Genética Médica donde el paciente ha sido tratado y previo consentimiento informado. 


\section{Referencias}

1. Bravo-Oro, A. Diagnóstico e intervenciones terapéuticas en la enfermedad de Pompe. Rev Med Inst Mex Seguro Soc. 2013; 51(5): 536-51

2. Byrne, BJ; Kishnani, PS; Case, LE; Merlini, L; MüllerFelber, W; Prasad, S; et al. Pompe disease: design, methodology, and early findings from the Pompe Registry. Mol Genet Metab. 2011; 103(1):1-11.

3. Jeong-A, L; Lishu, L; Nina, R. Pompe disease: from pathophysiology to therapy and back again. Front Aging Neurosci. 2014; 6(177).

4. Ans, T; Van der Ploeg, M; Clemens, P; Corzo, D. A Randomized Study of Alglucosidase Alfa in Late-Onset Pompe's Disease. N Engl J Med. 2010; 362:1396-1406.

5. Rare Disease Act 2002. An Act to amend the Public Health Service Act to establish an Office of Rare Diseases at the National Institutes of Health, and for other purposes. November 6, 2002.

6. Cortes, F. Las enfermedades raras. Revista médica Clínica Las Condes. 2015; 26(4): 425-431.

7. Chien, Y; Hwu, WY; Lee, NY. Pompe Disease: Early Diagnosis and Early Treatment Make a Difference. Pediatrics \& Neonatology. 2013; 54(4); 219-227.

8. Sepp, E; Reuser, A; Lohi, H. A Nonsense Mutation in the Acid $\alpha$-Glucosidase Gene Causes Pompe Disease in Finnish and Swedish Lapphunds. PLOS ONE 2013.

9. Van, Carla. Newborn screening for pompe disease? a qualitative study exploring professional views. BMC Pediatr. 2014; 14(203)

10. Leslie, N; Tinkle, BT. Glycogen Storage Disease Type II (Pompe Disease). University of Washington, Seattle. 2007
11. Cupler, EJ; Berger, KI; Leshner, RT. AANEM Consensus Committee on Late-onset Pompe Disease. Consensus treatment recommendations for late-onset Pompe disease. Muscle Nerve. 2012; 45:319-33.

12. American College of Medical Genetics. Pompe disease diagnosis and management guideline. Disponible en http://www.nature. com/gim/journal/v8/n5/abs/gim200650a.html. 2006.

13. Weinreich, SS; Rigter, T; Van El, CG; Dondorp, WJ. Public support for neonatal screening for Pompe disease, a broadphenotype condition. Orphanet J Rare Dis. 2012; 7(15).

14. Ross, LF; Waggoner, DJ. Parents: critical stakeholders in expanding newborn screening. J Pediatr. 2012; 161(3):385-9.

15. Dubrovsky, A; Fulgenzi, E; Amartino, H; Carlés, D; Corderi, J; De Vito, E. Consenso argentino para el diagnóstico, seguimiento y tratamiento de la enfermedad de Pompe. Neurología Argentina. 2014; 6(2): 96-113.

16. Han, S; Pope, R; Li, S; Kishnani, P; Steet, R; Koeberl, D. A beta-blocker, propranolol, decreases the efficacy from enzyme replacement therapy in Pompe disease. Molecular Genetics and Metabolism. 2016; 117(2):114-119.

17. Nausa, J. G. (2014). "Evaluación Clínica y radiográfica de injertos biocerámicos tipo Hidroxiapatita como alternativa en la reconstrucción de alveolos dentarios postexodoncia."

18. Millan, J. and L. Yunda (2014). "An Open-Access Web-based medical image atlas for collaborative Medical image sharing, processing, Web Semantic searching and analysis with uses in medical training, research and second opinion of cases." Nova 12(22): 143-150. 